\title{
Scaling Spatial Transformation: Smart Specialization of Urban Capabilities in the Helsinki Region
}

\author{
Renita Niemi, Eelis Rytkönen, Robert Eriksson, and Suvi Nenonen
}

\author{
"A great city is not to be confounded with a populous one. ") \\ Aristotle (384-322 BC) \\ Philospher and scientist
}

\begin{abstract}
Societies are shifting towards more complex structures and agile networks through spatial transformation. That shift affects the ways in which citizens interact with and within their physical and virtual surroundings. The interactions define purposes for the modern hybrid spaces, depending on individual demands in relation to space and time. As facilities per se are becoming less relevant, spatial concepts and service that support, attract, and engage modern individuals must be invented. The capabilities of user-orientated processes are important in terms of connectivity, co-creation, and communication, involvement in change, and control as well as governance. This article explores the potential scaling in diverse spatial transformations and summarizes the lessons learned from managing a campus as a small city to managing a larger-scale urban area. The study uses a case study methodology: the data was collected through interviews and document analysis. The framework of five urban capabilities (5Cs), which were initially introduced by the urbanist John Worthington, guided the content analysis of data. The results indicate that the lessons learned in the diverse urban projects can be scaled from a minor urban-area campus to a large urban area. Users of spaces have a need and will to collaborate, co-create, and impact their environments. This view expands the roles of decision makers and planners to controlling the uses of spaces for supporting grassroot initiatives. Consequently, active citizens engage and contribute, which can be a driving force for co-creation, shared ownership, and attractiveness of small- and large-scale areas.
\end{abstract}

\section{Introduction}

The digital paradigm and technological innovations are changing the way we interact with and understand our surrounding spaces. Castells (2004) argues that we are shifting from a space of static places to a space of flows where information and knowledge are exchanged globally in ever-denser networks. Building on similar thoughts, Mehaffy (2014) sees cities through the lenses of six fundamental elements: cities as spatial networks, as social networks, as partially decentralized and as partially generated by self-organizing agents, as partially scale-free, as partially scale-dependent, and as cognitive and symbolic systems. Nonaka $(1998,2000)$ introduces the concept of $\mathrm{Ba}$ in organizational settings as a shared physical, social, and virtual space that can facilitate knowledge creation between individuals. Multiple scholars argue that, as the cycles of change become ever denser in increasingly competitive markets, the capability of creating adaptable built environment is becoming more and more crucial (Finch, 2012).

However, the essence of moving from the setting of a stable built environment towards dynamic multi-locational concepts is a major factor in scalable urban development. Nevertheless, the mere concepts do not suffice: the change in user behaviour and community culture is an essential driver of the emerging change. Increasing the alignment and integration of virtual and physical environments has induced new forms of behaviour. 


\title{
Scaling Spatial Transformation: Smart Specialization of Urban Capabilities
}

\author{
Renita Niemi, Eelis Rytkönen, Robert Eriksson, and Suvi Nenonen
}

Large parts of our daily activities take place in virtual environments and affect the physical layers of our environment. For example, Demos Helsinki (2014) announced a Smartup Manifesto listing organizations that represent a new wave of startups that focus on increasing the efficiency of using physical resources by offering virtual services, such as AirBnB, Uber, Sharetribe, and Venuu. As another simple example, one can observe the disappearance of telephone booths from cities and buildings as static small cubicles - now the telephone is a mobile, intangible bubble around us wherever we are. Diverse solutions, propositions, and recommendations are available for mobile phones. Moreover, the city of London, for example, has updated numerous old booths to providing free Wi-Fi access. Physical, social, and virtual layers of our environments are inevitably more and more integrated.

Mobility has increased individual freedom and choice. Sustainability drivers have made individuals more aware of, for example, different transportation alternatives. New ways of using and sharing resources are increasing (Brinko et al., 2014; Lindsay, 2014; Termaat et al., 2014) due to the rise of the sharing economy. Additionally, the emphasis on social sustainability includes issues such as happiness, wellbeing, and satisfaction with life. The flexibility and attractiveness of the social, virtual, and organizational infrastructures that a city creates provides a competitive edge. The greater variety cities are able to offer in terms of local, dense, and thematic communities, the larger their potential to attract talented people and organizations. The aim of this article is to explore the potential of scaling in diverse spatial transformations. The research question asked is whether the practices of managing a campus as a small city can be scaled to managing a larger-scale urban area.

\section{Drivers of Change}

The consequences of more mobile lives and work styles can be seen in academia and the private sector: both are struggling with low space-utilization rates resulting in high bills. Multiple studies in European and USbased universities indicate space-utilization rates of less than $40 \%$ during the office hours (Den Heijer, 2011; Den Heijer \& Zovlas, 2014; Harrison \& Les Hutton, 2014; Neary et al., 2010; University Herald, 2013). According to Den Heijer and Zovlas (2014), campuses constitute about 5-15\% of European university budgets.

For example, a recent study in Aalto University showed that space-utilization rates tend to vary between
20-40\% during office hours (Hietanen, 2014). Aalto University's main campus consists of 30 buildings covering an area of about 240,000 square meters. The campus costs, including rents and maintenance, exceeded 70 million euro in 2015. Facilities form the second largest cost after human resources. At the same time, despite slightly higher utilization rates, a million square meters of office premises lack tenants in the metropolitan area of Helsinki. These vacancies represent about $12 \%$ of the total office building mass in Helsinki and $20 \%$ in nearby Espoo. These empty or half-empty offices are part of the image of the campus and cities. The supply of the built environment does not match the demands of mobile life and work.

It seems that the places where knowledge work is accomplished are scattered across multiple spaces, from traditional offices and business park complexes, to hubs, co-working spaces, and home offices (Waber et al., 2014). What we used to know as the "third place" that supports the infrastructure created by offices and homes are remodelled to diverse service offers in a more conscious way (e.g., Brinko et al., 2014; Termaat et al., 2014). The organization no longer defines the location of the work; the work is disseminated all over the city structure: homes, public spaces, the premises of clients or partnering organizations, private cafes and restaurants, and diverse co-working places. The whole city can be seen as an office and in the minor scale, and similar dissemination can be seen in the campus area: the location of an individual's own department or faculty is no longer the main determinant. The administrative section can have an address, but networks rarely have a stable address - learning and working on the campus occurs in diverse locations if the university offers the mobile possibilities.

The total amount of square meters per knowledge worker is not thus diminishing, but probably even increasing. Even though workplace changes from traditional office concepts towards activity-based concepts, offices can reduce the amount of square meters in relation to one workstation: the amount is increasing per employee, because mobile work can be completed in diverse work zones. Work is scattered across multiple places and dictated by the collaborative processes - considering the utilization rate of diverse places is much more relevant than the rate of one single workstation. A similar trend can be identified in the context of learning environments. The use of classrooms is no longer the main success factor but greater emphasis should be put on the amount, quality, diversity, and use of learning and working zones - to scatter the learning and work- 


\section{Scaling Spatial Transformation: Smart Specialization of Urban Capabilities}

Renita Niemi, Eelis Rytkönen, Robert Eriksson, and Suvi Nenonen

ing activities around the campus instead of the silos of faculties, or building wings for staff or students only.

The dominant role of institutional ownership of buildings and individual ownership of desks will most likely diminish in the course of time. The booming trends indicate that work is increasingly accomplished in the shared premises of multiple organizations that have a common agenda. Gathering the stakeholders and facilitating their collaboration requires operators who would take the premises into efficient use. This approach will most likely offer new business opportunities while changing the dynamics of the traditional ways of leasing spaces on the basis of fixed contracts for multiple years. Dynamic spatial abilities, such as flexibility and adaptability of the building services and processes that building facilitators and operators are offering, will probably play an increasingly important role in the market.

Actually, new operators are constantly entering the market and diverse concepts can subsequently be identified both in the city and on the campus. Examples of multi-locational work concepts in the metropolitan area of Helsinki include service concepts in co-working such as Kontoret, Hub13, Urban Office, Urban Mill, and StartUp Sauna, to name a few. Kontoret as a concept aims to build a network of on-demand spaces for modern knowledge workers. The operators of Urban Mill strive to replicate the lean methodology they applied in Urban Mill and take over underutilized assets beyond the campus in an attempt to attract organizations and create more thematic communities that would benefit from a common platform. In their operations and risky business strategy, facilities management is in the secondary role, and greater emphasis is put on the community management role, which is supported by physical and virtual infrastructures.

The focal question for both effective and efficient workplace orchestration lies in scalability: from office space to the use of the building to the use of the city. On the space-user level, the core is thus in the scalability of the new ways of working and learning at individual, team, and organizational levels. On the other hand, there are varieties of reasons why organizations do not support the dissemination of work. Lindsay (2013) proposes that co-working generally falls into one of the three categories: co-working in a separate location, co-habiting a common space with a partnering organization, or opening up an organization's workspace to a wider community, resulting in a working commons. Co-working in a separate location involves shared environments where individuals and small groups gather together to work in a community, usually paid for on a membership basis and invoiced either monthly or daily. These spaces provide a community workspace with shared services that let individuals and small groups share ideas and mutually support each other's work. Lindsay (2013) has found that corporate organizations are encouraging their own employees to work in co-working spaces as an alternative to their regular workspace, not primarily to save on costs, but to facilitate their interaction and knowledge sharing with others, and to inspire creativity.

In addition to co-working spaces, organizations are opening up their own workspace to a wider community in an attempt to invite others in to share it (Lindsay, 2013). The working commons emerges as one kind of a semi-public shared space similar to the learning commons in the university context. University campuses have moved away from libraries exclusively designated as places for reflective study, to spaces where informal and ad hoc collaboration happens in learning commons. The Aalto University library is following this direction in developing its premises through bottom-up processes such as AaltoHUBs, which recycles underutilized spaces through collaborative, community-engaging design processes. Typically, these spaces include places to meet, study, make connections, and exchange ideas. Food and drink are welcomed, furniture and equipment are mobile or reconfigurable, and access is allowed at all hours. The settings of the space change by the hour, day, and week. Municipal governments could have an emerging role in hosting these kinds of shared spaces.

Co-habiting means several partnering organizations sharing a common work environment. They are types of workspace where, rather than an individual organization opening up to others or to the wider community, several organizations together share a work environment with the purpose of gaining from each other's knowledge and experience. Furthermore, Lindsay (2014) has identified six new types of workspaces that are supplanting the corporate campus because they offer ways to increase the probability of interactions that lead to innovation and productivity: real-time offices, permeable offices, office networks, office neighbourhoods, office-as-a-service, and the new guilds.

The complex environment challenges municipal decision makers and politicians to prioritize and make decisions among a vast number of initiatives, projects, and events. Organic bottom-up projects have become 


\section{Scaling Spatial Transformation: Smart Specialization of Urban Capabilities}

Renita Niemi, Eelis Rytkönen, Robert Eriksson, and Suvi Nenonen

more and more attractive alongside hierarchically structured top-down projects. In a recent study (Rytkönen, 2015), the same phenomenon has been detected in micro-scale in university campuses, where spatial transformation is affecting the rules of the game. From the university campus management organization, the spatial transformation seems to require the ability to balance between individual and communal demands, local and global foci, and project-based pioneer projects and standardized hierarchical projects. In order to support the core tasks of universities, campus managers and university administrators should support multiple business models and processes, thereby enabling interdisciplinary, cross-organizational actions to take place on their campuses. Rather than facilitating or managing the facilities per se, the essence of their work seems to shift towards orchestrating the communities that act within the facilities.

On the scale of urban planning, Horelli and Wallin (2013) have similarly identified that, rather than only having roles as administrators and hierarchical watchdogs, the tasks of city managers and planners are expanding towards following, engaging, empowering, and supporting the grassroot pioneer initiatives that attract interest and buzz in the cities. Balancing between them and the more stable, standardized, and static processes is a focal task in competing in the global market. On the one hand, it is important to identify the typographies of different scales in order to respond to the needs of mobile living, working, and learning; but, on the other hand, it is important to identify the common factors in diverse processes of developing such a physical and virtual infrastructure.

\section{Methodology}

This study took a qualitative approach in an attempt to build propositions on data collected about the case study. The case is urban area consisting of three districts: the university main campus as a district for science, research, education, and arts; the business district; and the cultural, living, leisure, and retail district. The content analysis of data was guided through the framework of five urban capabilities, which were initially introduced by the urbanist John Worthington (Worthington \& Bouwman, 2012). Allowing comparison between the approaches of six learning cities' projects, discussions were structured around these five themes, which are the "5Cs": connecting, changing, collaborating, communicating, and controlling. The 5Cs have been further explored and developed by Niemi and colleagues (Mangs et al., 2013; Niemi et al., 2013), who con- cluded that the 5Cs framework can be applied to the analysis of open-ended projects with clear goals, budgets, and deadlines spread over time (Niemi et al., 2013). The approach was furthermore seen as scalable in the city and in district scales, and particularly for observing certain everyday practices. Although the scholars pointed out its limitations as an evaluation tool, they emphasized its ability to recognize different phenomena in city development.

\section{Case Study: Smart Specialization in the Helsinki Region}

History, visions, decision making, and physical dimensions The so-called T3 area of Espoo consists of three districts: the Aalto University main campus as a district for science, research, education, and arts; the business district of Keilaniemi; and the cultural, living, leisure, and retail district of Tapiola. Each district has a rich history: the task of the city is to integrate the original Tapiola garden city vision from the 1960s, the Keilaniemi business tower vision from the 1990s, and Otaniemi campus vision that was updated from the original 1960s vision of Alvar Aalto, to the 2006 vision for Otaniemi as a hub for science and business, and most recently, the interdisciplinary Aalto University campus vision of 2011, bringing together arts, technology, and business. Together, these districts form one of the most attractive areas to live and to do business globally. However, in order to make it even more attractive, professional operators are needed to facilitate and integrate collaboration that creates synergies. The region and its districts are illustrated in Figure 1. The figure identifies the three different districts found in the T3 area. The five capabilities are covered throughout these districts and can be found more intensely unified in the minuscule scale in the Science and Technology district, which relates to the campus.

The Aalto University main campus, representing one of the three districts of the T3 area, is the playground for the university that merged in 2010 from three original universities: the Helsinki University of Technology, the University of Art and Design Helsinki (TaiK), and the Helsinki School of Economics. The vision of Aalto University, and consequently, the vision for its campus, is strongly rooted in the interdisciplinary synergies between technology, arts, and business, and it has been collaboratively created by 2500 Aalto community members. It aims to be a world-class university by 2020 . In order to facilitate the synergies, the majority of the actions are centralized on the main campus of the former Helsinki University of Technology. The former TaiK 


\section{Scaling Spatial Transformation: Smart Specialization of Urban Capabilities}

\section{Renita Niemi, Eelis Rytkönen, Robert Eriksson, and Suvi Nenonen}

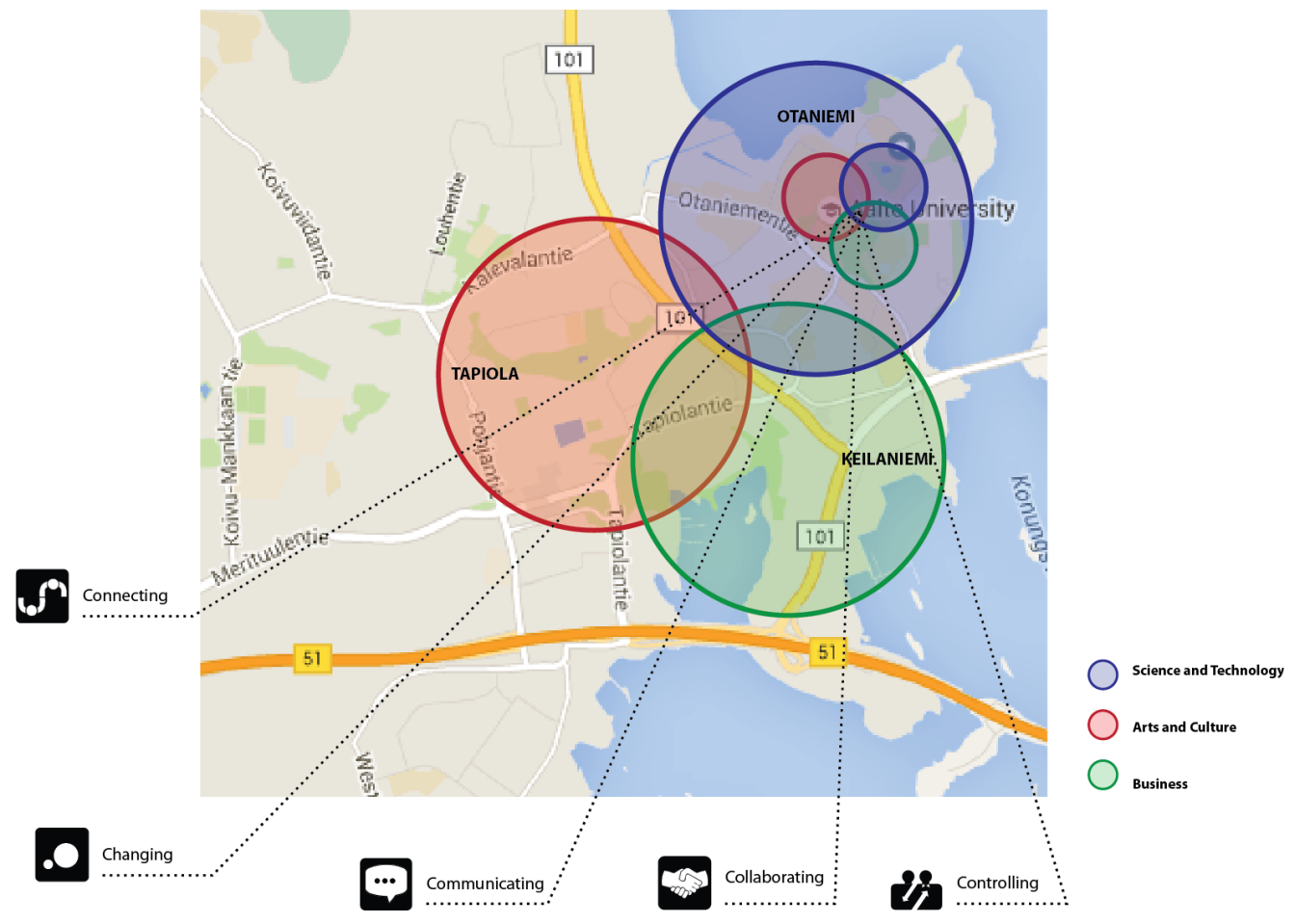

Figure 1. The T3 region and the 5Cs model (applied from Niemi et al., 2013; Worthington \& Bouwman, 2012)

campus on the other side of the Metropolitan area of Helsinki is abandoned, as a new construction is being built on Aalto, the main campus and all the bachelorlevel education is centralized to the bachelor cradle, formerly the main building of the Helsinki University of Technology. In addition to these and other strategic decisions, various grassroot, bottom-up projects are ongoing: the alternative learning and research environments such as the Design Factory, StartUp Sauna, AaltoHUBs, ADDlab, and Urban Mill, to name a few. Their quickand-dirty, iterative approaches to creating attractive collaboration seem unique in the university context and have the potential to scale up to the urban development level (Rytkönen et al., 2014).

\section{Results}

The results indicate that the lessons learned in the diverse urban project can be scaled from a minor campus area to a large urban-area scale. Users of space have a need and will to collaborate, co-create, and impact their environments. This view expands the roles of decision makers and planners from controlling the uses of spaces to supporting grassroot initiatives. Consequently, active citizens engage and contribute, which can be a driving force for co-creation, shared ownership, and attractiveness of small- and large-scale.
Theme 1: Connecting - area and people

Connectivity refers to the connection between different communities as well as to the capability to connect to the physical environment, with the help of virtual infrastructure such as social media and social networks. Aalto University has three separate campus areas that will be diminished to two: one in the Helsinki city centre and one in the traditional campus location in Espoo, in the former campus of the University of Technology. The vision of Aalto University is to connect the professionals of arts, technology, and business. Both campuses have their own buildings although concepts such as the Aalto Design Factory and Learning Hubs are the elements truly connecting Aalto University. These places are physical surroundings and virtual platforms; they are diverse ways of accomplishing tasks processes to develop, use, and maintain places that serve as platforms for collaboration between different disciplines and actors.

The T3 area has three urban areas with different profiles to connect: the Aalto University campus as an area for research and education, the business district of Keilaniemi, and the cultural, living, leisure, and retail district of Tapiola. Large highways physically separate three districts. The main driver of the connection is often viewed to be transportation. The T3 development 


\title{
Scaling Spatial Transformation: Smart Specialization of Urban Capabilities
}

\author{
Renita Niemi, Eelis Rytkönen, Robert Eriksson, and Suvi Nenonen
}

combines different modes of transport, and the new metro line is the main connector. It is seen as a major link between three areas as well as linking the region to the city centre of Helsinki. Additionally, the connecting characteristics of nature are identified in terms of cycling, walking, and using natural pathways and green corridors as shortcuts across the areas. Water as an element has more surrounding than connecting characteristics. Connectivity can be encouraged by creating hotspots (i.e., a physical location with wireless access), which are connected to each other as a network of places as processes in order to co-create, operate, and co-develop them. However, communities require active facilitation. Even though packing interdisciplinary and cross-organizational students, researchers, professors, practitioners, and people from the public sphere into a dense area might support connectivity due to proximity, it is not enough if there are no processes to connect the diverse actors. Allen and Henn (2008) argue that increasing the opportunities for knowledge transfer, inspiration, and later innovation is achieved in organizations by maximizing the opportunities for communication. This concludes the configuration of the organizational structure and physical space. Similarly, a connector in infrastructure does not guarantee connectivity in the social context if it does not serve mobility between the areas, which also connect people through the processes of creating the area.

\section{Theme 2: Changing - towards the vision}

Change is a natural phenomenon of development, but the essential aspect in organizational settings is in reacting to change. Change occurs both physically and perceptually, and it is more about changing a mindset than physical alterations per se. The current changes on the Aalto University campus are based on the organizational change of the university, which affects the built environment by and large. Innovative grassroot initiatives are blossoming next to massive traditional renovations, new investments, and exits. Larger change nurtures smaller change and vice versa. However, the cultural change of breaking out from the traditional silos takes time, and so does engaging the middle management at the core of continual change. The organizational change of Aalto University has potential to affect the city of Espoo as an attractor of new types of businesses to the Keilaniemi area.

The challenge in Aalto University is to integrate three old institutional systems while respecting the original identities and creating motivators for the units to follow and implement the ambitious new visions. The same challenge applies to the T3 area. From these ingredi- ents, a believable synthesis and incentive system, that the actors from all areas can relate to and are motivated to implement, should be created. Both Aalto University and the T3 area could become more resilient and adaptable to change from spatial, organizational, and operational perspectives. Involving people in the early phase of the development process decreases the unwillingness for change. Flexibility and resilience are the focal capabilities in recovering from the changes. The resilience strategy for the T3 area could be part of the visionary work conducted in long-term urban development.

\section{Theme 3: Communicating - narrative and image of the area}

Communication concerns promotional activities and interaction with others. By means of communications, a brand and a collective image can be built, but individuals build identity. A brand can be seen as a collective agreement of the image, whereas identity concerns an individual, their self-perception and self-presentation expressing one's personality. The Aalto University brand is strong and externally well known. The Aalto main campus offers world-class examples of co-creational actions and initiatives that have been well-communicated and function as communication platforms for their user communities. Yet, these communities only represent a small portion of early adopters among the university actors. The internal institutional units of Aalto University are still heavily struggling with building the Aalto identity, which is why the internal communications require greater investment of money and time. AaltoHUB is one of the projects that aim to affect the overall identity of Aalto people. The hubs are co-created, informal places for studying - they offer relaxed and flexible learning environments where the students need them the most. The challenge in the T3 area is communication between three districts. Today, its role is undervalued. In the future, it will be central to the success of the whole area. The city of Espoo should engage its citizens and empower entrepreneurs and industries to follow the external communications examples of Aalto University. Arnstein (1969) introduced an idea of citizen participation as citizen power.

There is no single sign to brand or identify the T3 area it is rather an internally strong mental model. To make it visible, sensible, and encouraging requires action on the physical and virtual, internal, and external communication channels. The capability to create experiences - the sense of the place and diversity - is increasing in Aalto University. This is important competence, because often decisions are based on feelings and, therefore, it is important that those involved in planning 


\section{Scaling Spatial Transformation: Smart Specialization of Urban Capabilities}

Renita Niemi, Eelis Rytkönen, Robert Eriksson, and Suvi Nenonen

processes are aware of this phenomenon and create (communicate) solutions in the best possible ways to support the end-user choice. The essence of successful grassroot activities is in the interaction and communications between the top management and grassroot actors. Two-way communication (top-down and bottom-up) is a process in which participants create and share information to research mutual understanding. Communications can raise awareness and change perceptions to support cultural, behavioural, and physical change.

\section{Theme 4: Collaborating - user involvement}

Collaboration means capability to collaborate informally and formally. Two key phenomena related to collaboration are complexity and diversity. Collaboration should not be seen as restrictive practices, but rather as a set of processes for creatively balancing conflicting and mutual interests. It is about working across different scales, interests, functions, and cultures with the aim of building up a community spirit. Collaboration includes both informal and formal processes.

The merger of three universities into one organization multiplies the complexity of the main Aalto campus. On the one hand, complexity forms a barrier to the new organization to collaborate internally. On the other hand, the increasing diversity offers great opportunities, which should not be underestimated. Due to the diverse characteristics of regional development projects in the T3 area, one can encounter questions that are "wicked", "messy", and "fuzzy" (Rittel \& Webber, 1973; Buchanan, 1992). One profession or industry simply cannot solve them alone, which is why collaboration is needed to overcome the issues of complexity and diversity. Aalto University plays an important role in creating partnerships and in linking and forming platforms for public and private sectors. One of the most promising concrete grassroot initiatives is Urban Mill, which focuses on facilitating new public-private-people partnerships in an attempt to find common and shared value within the public and private sectors. On Aalto's main campus, interdisciplinary and cross-organizational collaboration has been fostered through these kinds of boundary objects. Buildings and spaces function as thematic platforms for collaboration around the campus, such as the Energy Platform, the Digi Platform, and the Living+ Platform. Creating a collaborative culture across organizations and disciplines requires time. However, a collaborative culture can be empowered by hybrid operators who facilitate the activity process. Aalto University has been successful in external collaboration, but the internal collaboration would require even more incentives to be reinforced - this is not the tradition of rewards systems in universities in general as they rather focus on individual merits and achievements.

In order to create a collaborative culture among the T3 spectrum of areas - culture, arts, living, leisure, business, and science - initiatives exist, including Espoo Innovation Garden, Espoo Day, and Base Camp, among others. These refer to innovative, common ways of working and a culture of collaboration and co-creation. The collaboration should be active among operators who identify and facilitate the collaboration of organizations and institutions with the same agenda or theme. This active facilitation could mentally draw areas closer to each other in an attempt to blur the physical boundaries. A continuous series of small events is essential to gradually raise awareness and change perceptions. When building a community, it can help to have engaging individuals who are willing to be pro-active and responsible and who then spread a climate of confidence and opportunity for change - they help in achieving a paradigm shift.

\section{Theme 5: Controlling - direction, principles, and rules} Controlling in this context should not be perceived as traditional top-down restrictive action. Rather, it is defined here as a continuous management process that has a forward-looking attitude. Control can be achieved through common direction, principles, and rules. Organizations should, therefore, be motivated to relate to and engage in the same principles. Successful control requires a balance between creating and reinforcing vision and mission, and then managing the process of change through a combination of regulatory controls and behaviour.

Aalto University has a strong mission and vision but the incentives for implementing them are contradictory. A path should be selected that either aims for high international university rankings with the criteria of interdisciplinary work and focus on societal impact or that position the organization in more traditional rankings emphasizing the merits of academic research. The lesson of successful bottom-up cases in Aalto is that shared control and active communications between top, middle, and bottom levels of organizations is important.

The vision of the T3 area for connecting the physically separated cultural, business, and science districts together challenges organizations to perform a profound cultural change and institutional collaboration. To perform such a change, boundary objects and thematic entities are needed - platforms that foster sharing across 


\title{
Scaling Spatial Transformation: Smart Specialization of Urban Capabilities
}

\author{
Renita Niemi, Eelis Rytkönen, Robert Eriksson, and Suvi Nenonen
}

organizations and nodes. The implementation can then be built on the ongoing collaboration. The developments of area management and areal operators evolve alongside the developments of the physical environment and the temporal development and control. Control can be managed through functioning partnerships between the key stakeholders with the agreed goals. The essence is in finding an operator with the ability to keep the different parties aligned and engaged. The operator has to orchestrate the process of change and sustain other stakeholders who are committed and keep the project alive. It is crucial to engage the actors to contribute to the common mission through incentives.

Reflecting on the learnings from the campus organization, it seems that pioneer facilities and community operators should start actively seeking underutilized facilities and start gathering stakeholders that benefit from one another under the same roof. The social and economic benefits from moving towards a more mixeduse, live, work, play society are the opportunity for knowledge distribution, local economic growth, and walkable neighbourhoods. On Aalto campus, the first steps have usually been thematic events and workshops. Moreover, these operators tend to offer pop-in and collaborative spatial entities where the organizations should not stay statically but dynamically spend some of their time collaborating with each other and then again head towards their clients or home organizations to share the lessons. It is yet remarkable that most of the campus real estate mass is still operated rather conservatively. To implement the novel and dynamic kind of culture on a larger scale takes time but the next generations are already knocking on the doors of the job market. The spatial transformation paradigm seems to be bubbling on the surface of the city as a blurry playfield for a mixture of working, learning, retail, leisure, and daily routines.

\section{Takeaways}

Based on our analysis of this case, we offer the following key insights:

1. Dynamic connectivity can be created through alternative spatial platforms and processes including cocreation, maintaining, and co-development.

2. Change has a pearl in it - incentives towards the targeted change and respect of minor-scale changes can provide elements for large-scale changes, too. Resilience can be a strategy for overcoming the ongoing turbulence of change.
3. Communication materializes in visible artifacts and in social discourse: a brand can be strong but identity weak - balancing between internal and external communication is as important as balancing between vertical and horizontal communication.

4. Collaboration is rarely linear - it happens even though it would seem chaotic, unclear, fuzzy, or wicked.

5. Control is about communication and incentives motivation cannot be commanded but ownership and empowerment can be enforced.

\section{Conclusions}

The shift in the concept of space from being a space of static places towards a more dynamic space of flows is evidently ongoing, as Castells (2004) and Nonaka (1998, 2000), among others, describe. As the activities increasingly mix, regardless of the space, the current practices in the built environment do incompletely support this mixture but tend to silo each activity in their own block. The 5C analysis indicates that the campus areas can function as great living labs for experimenting and prototyping bottom-up concepts for facilitating collaboration among public and private stakeholders as they are densely packed in a manageable entity and as universities create new models and practices, through their core business, research. They are also rather objective and capable of providing a common ground for institutes, municipalities, decision makers, politicians, business, and industry representatives alike.

The tested solutions can thereafter be applied on a larger city scale to answer demands outside the university barriers. Based on the results of the analysis, it seems clear that the spatial solutions are only knots in the network. Collaboration needs to reach beyond the single hotspots in order to create an interactive network where great minds interact in the spaces of flows. To truly reach the business potential of mobile knowledge work facilitation and revitalize larger city areas, we need new types of hybrid operators - or new processes, practices, and businesses for the existing operators that are capable of strengthening what is in between the knots. The buildings themselves are not in the core but the essence is in managing what happens inside and in between them, in the network facilitation.

The challenge in land use and planning is that law dictates it, and there is no control or ownership of the management of processes - a situation similar to the 


\section{Scaling Spatial Transformation: Smart Specialization of Urban Capabilities}

\section{Renita Niemi, Eelis Rytkönen, Robert Eriksson, and Suvi Nenonen}

campus area only a decade ago. Strong individuals who seem to have strong ownership of the projects lead the case examples. If they leave the organization, they are difficult to replace and the successful initiatives might discontinue. On the other hand, little by little, these novel practices and niche innovations build on each other, creating change in the standards of processes. In order for the bottom-up processes to take place by and large in the built environment, the approach of managing and commanding through hierarchies, standards, and mechanisms of passive control must be flipped to the approach of actively orchestrating the actions by support, incentives, and other enablers. Furthermore, the results must be measured in terms of the holistic quality of the action and the effectiveness that the built environment enables - not solely in terms of the efficiency of the built environment itself.

\section{About the Authors}

Renita Niemi is a design strategist for humancentered change. She is a creative professional who graduated in 2004 from the University of Art and Design Helsinki (TaiK) in Finland. She has major in Industrial and Strategic Design and minor in International Design Business Management (IDBM). Currently, she focuses on her part-time $\mathrm{PhD}$ studies at Aalto University. Having several years of academic background working at TaiK and Aalto University, she is experienced at multidisciplinary research, concept development, and design. She has completed a diverse range of design research projects related to housing and living. Her ambition is to form broad initiatives to facilitate further innovation, which involves networking between people with different perspectives, as well as rapid experimentation and creative collaboration. Her passions are greatly connected to planning, design, services, and innovation in context of the urban environment.

Eelis Rytkönen is interested in socio-technical phenomena, managerial practices, and spatial design in organizational settings. Currently, he works as a researcher for the BES research group in Aalto University, Finland, where he is finishing his doctorate research on dynamics of campus management in spatial transformation. He holds a BSc in Real Estate Economics and an interdisciplinary MSc degree in International Design Business Management (IDBM).

Robert Eriksson graduated as an architect from Chalmers University of Technology in Gothenburg, Sweden, in 2005. Currently, he works as a senior advisor in a consultancy solving problems related to the built environment. He has extensive experience in city planning, renewable energy, and open data. Robert has also been active in research, having published scientific publications related to, for example, user empowerment and future campus development.

Suvi Nenonen has worked as a Research Manager in Aalto University, Finland, since finishing her doctorate in 2005 on work environments that support new knowledge creation. She has published over a hundred scientific publications and acts actively in multiple international facilities management networks such as EuroFM and NewWOW. Suvi also acts as a docent in the Tampere University of Technology, Finland. 


\section{Scaling Spatial Transformation: Smart Specialization of Urban Capabilities}

Renita Niemi, Eelis Rytkönen, Robert Eriksson, and Suvi Nenonen

\section{References}

Allen, T. J., \& Henn, G.W. 2008. The Organization and Architecture of Innovation. Oxford, UK: Elsevier.

Ariely, T. 2008. Predictably Irrational the Hidden Forces That Shape Our Decisions. New York: Harper Collins.

Arnstein, S. R. 1969. A Ladder of Citizen Participation. Journal of the American Institute of Planners, 35(4): 216-224. http://dx.doi.org/10.1080/01944366908977225

Buchanan, R. 1992. Wicked Problems in Design Thinking. Design Issues, 8(2): 5-21. http://dx.doi.org/10.2307/1511637

Brinko, R., Van Meel, J., \& Nielsen, S. B. 2014. The Shared Building Portfolio: An Exploration and Typology. In Proceedings of CIB Facilities Management Conference 2014: 154-165. Copenhagen: Technical University of Denmark.

Castells, M. 2004. Space of Flows, Space of Places: Materials for a Theory of Urbanism in the Information Age. In S. Graham (Ed.), The Cybercities Reader: 82-93. London: Routledge.

Demos Helsinki. 2014. Smartup Manifesto - A New Wave of High Impact Startups Is Emerging from Consumer Cleantech. Helsinki: Demos Helsinki.

Finch, E. (Ed.) 2012. Facilities Change Management. Chichester, UK : Wiley-Blackwell.

Hietanen, P. 2014. Co-designing Campus. Seminar presented at the Nordic Association of University Administrators (NUAS) on August 26, 2014. Available at:

http://blogs.helsinki.fi/nuas-facilities-and-environment2014/files/2014/08/Co-designing-campus_Paivi-Hietanen.pdf

Horelli, L., \& Wallin, S. 2013. Towards an Architecture of Opportunities. In L. Horelli (Ed.), New Approaches to Urban Planning - Insights from Participatory Communities: 153-161. Helsinki: Aalto University.

Lindsay, G. 2013. Coworking Spaces From Grind to GRid70 Help Employees Work Beyond the Cube. Fastcompany, February 11, 2013. Accessed October 15, 2014:

http://www.fastcompany.com/3004915/coworking-nextspace

Lindsay, G. 2014. 6 New Workspaces That Are Killing the Corporate Campus. Harvard Business Review, September 18, 2014. Accessed October 15, 2014:

http://blogs.hbr.org/2014/09/6-new-workspaces-that-are-killingthe-corporate-campus/
Mehaffy, M. 2014. What Can a 'Science of Cities' Offer Planners? Planetizen, June 25, 2014. Accessed October 15, 2014: http://www.planetizen.com/node/69957

Niemi, R., Nenonen, S., \& Junnonen, J-M. 2013. Investigating the Competencies for Serviceability of Urban Areas. Paper presented at the CIB 2013 World Congress, May 5-9, 2013. Brisbane, Australia.

Nonaka, I., \& Konno, N. 1998. The Concept Of "Ba": Building a Foundation for Knowledge Creation. California Management Review, 40(3): 40-54.

Nonaka, I., Toyama, R., \& Konno, N. 2000. SECI, Ba and Leadership: A Unified Model of Dynamic Knowledge Creation. Long Range Planning, 33(1): 5-34. http://dx.doi.org/10.1016/S0024-6301(99)00115-6

Mangs, A., Väistö, T., Karhu, J., Rajala, A., \& Niemi, R. 2013. Facilitating the Emergence and Development of Engagement Networks in Urban Surroundings. Paper presented at the Industrial Marketing and Purchasing (IMP) Conference 2013, August 30 - September 2, 2013. Atlanta, GA.

Rittel, H. W. J., \& Webber M. M. 1973. Dilemmas in a General Theory of Planning. Policy Science, 4(2): 155-169. http://dx.doi.org/10.1007/BF01405730

Rytkönen, E. 2015. University Campuses in Spatial Transformation: A Business Model Typology of Case Aalto University. Facilities, 33(9/10): 794-818. http://dx.doi.org/10.1108/F-04-2014-0042

Rytkönen, E., Nenonen, S., \& Österlund, E. 2014. Facilitating Interdisciplinary Learning Communities: A Case Study from Finland. In Proceedings of CIB Facilities Management Conference 2014: 354-368. Copenhagen: Technical University of Denmark.

Termaat, A., Van Sprang, H., \& Groen, B. 2014. Workspace 2020 Club, Hub and Co-work Space: Business Models for Optimal FM Support for Office Workers in the Future. Proceedings of the European Facility Management Conference (EFMC), 4-6 June, 2014. Berlin: EFMC.

Waber, B., Magnolfi, J., \& Lindsay, G. 2014. Workspaces That Move People. Harvard Business Review, 92(10): 68-77.

Worthington, J., \& Bouwman, H. 2012. Places of Connection - Themes, Concepts and Principles. Utrecht: The Academy of Urbanism; Learning Cities Platform. 